\title{
Requirement elicitation: Requirements conflict resolution and communication model for Telecommunication Sector
}

\author{
Mohammed Burhanur Rehman ${ }^{1}$, Hatim M. Elhassan Ibrahim Dafallaa ${ }^{2}$, Nazir Ahmad ${ }^{3}$, Iqrar \\ Ahmad $^{4}$, Mamoon Rashid ${ }^{5}$, Rizwan Khan ${ }^{6}$ \\ \{malrhman@kku.edu.sa ${ }^{1}$, hibrahem@kku.edu.sa², naahmad@kku.edu.sa ${ }^{3}$, iaazad@kku.edu.sa ${ }^{4}$, \\ mamoon873@gmail.com ${ }^{5}$,rizwan.khan87@hotmail.com ${ }^{6}$ \} \\ Dept. of information system Community College, King Khalid University Muhayel, Kingdom of Saudi \\ Arabia ${ }^{1,2,3,4}$ \\ Lovely Professional University, Punjab India ${ }^{5}$, Al-Barkaat College of Graduate Studies Aligarh India ${ }^{6}$
}

\begin{abstract}
Requirements elicitation (RE) phase is very critical and crucial to the success of IS projects in telecommunication sector. Unfortunately, this phase of IS projects development is susceptible to a large degree of errors, affected by key factors embedded in the applied communication techniques, which results in a requirement conflicts that widen the gap of what is being build and what is being desired by the stakeholders. The aim of this paper is to present a Requirements conflict resolution and communication model to address the lack of a systematic mechanism to quantify the communication obstacles and to classify the requirements conflicts in the RE phase. The proposed model will introduce the conflict detection and resolution mechanism based on the normalized cross correlations function (NCCF), standard deviation (SD) and the standard error (SE) functions to detect and quantify the conflicted requirements based on the calculation of the requirements correlations and accuracy. The proposed model will overcome the reoccurring issues of the requirement elicitation phase.
\end{abstract}

Keywords: Requirements elicitation, Communication issues, Requirements conflict.

\section{Introduction}

Communication is at the heart of requirements elicitation, for without effective communication between the stakeholder and the analyst, requirements will not be elicited properly [2]. As such Communicating requirements is significantly important to any successful implementation of software [3]. Communication as a model can be explained as the interchange, of the needs and wants of the stakeholders' community in an oral mold for a technology solution derived from the collected requirements. The large dependency of the elicitation process is placed on the human factor; which adds and undesired complexity to the requirement process. The technology community "requirement engineering" and the stakeholder are generally coming from a different background, mostly they bring different perspectives to the requirement elicitation process, the views of both requirement engineering and the stakeholder can be skeptically different. Thus, 
skepticality can blur the desired system definition because what is being captured by the requirement engineering will not reflect the stakeholder views of the system.

(Lyytinen and Hirschheim [4] categorized system failures into four types into four sorts: correspondence; process; collaboration; and desire. Barring the main type of failure from her investigation), (Macaulay [5] recognized five potential reasons for the three outstanding sorts of failures, displayed in order of affect: Poor correspondence among the individuals (process, communication, desire), absence of proper information or shared getting (collaboration, desire), improper, inadequate or off base documentation (connection, desire), absence of an efficient (procedure) and poor administration of individuals or assets).

\section{The Requirements Conflict Resolution and Communication Model}




Incomplete, inappropriate and conflict requirement has a vital role in the success of the requirement elicitation; there is a need to methodological approach to quantify these major issues that greatly affects the success or failure of requirements elicitation process to enable a higher level of shared understanding between the system analyst and the stakeholder for a better requirements outcome.

The current researches on the requirement elicitation issues focuses on different problem domains, and there is almost no unified framework to systematically guide the requirement engineer through the whole elicitation process. In the research studies, the importance of the proper communication and shared knowledge between the system analyst and the stakeholder were considered as vital but few solutions were provided for the problem, mostly coming from psychology and cognitive background. There is such a need to make sure that the gathered requirements have completeness sense and coverage of all the system or software aspects. Thus, there is a need to design a mechanism to quantify the communication obstacles arising in the course of the elicitation process and to detect and classify the requirements conflicts.

The proposed model Figure 1 will develop a more robust elicitation framework to help both the analyst and stakeholder in achieving a higher shared knowledge level through a proper communication repairs and produce a conflict free requirements that will satisfy the stakeholder needs in the intended system or software. A model that delivers complete, appropriate, clear, and a conflict free requirements, through refining and filtering process function.

The proposed model will help stabilizing the requirement elicitation process and contributes to the success of many projects.

\subsection{Model attributes}

The proposed model starts with a pre scan of the designated stakeholders to classify their technical background to facilitate the mean of communication language (formal / informal). The system analyst is more technical oriented; the use of formal technical language might not be understood by the stakeholder, which could cause a miscommunication (ambiguity). To overcome this shortcoming, the model pre scan classifies the stakeholders and selects the proper communication mode (formal / informal) Table 1, to be referenced by the analyst during the elicitation session.

Table 1. Stakeholder preference

\begin{tabular}{|l|l|}
\hline Stakeholder type & Category \\
\hline Stakeholder who is technical aware & Formal \\
\hline Stakeholder who is not technical aware & Informal \\
\hline
\end{tabular}




\subsubsection{Matching the methods}

The matching process links the elicitation technique to its fitting knowledge transfer method. The model will inject the selected elicitation technique in play with the proper transfer method selected from the pool as it stated in Table 2.

Table 2. Knowledge transfer method

\begin{tabular}{|c|c|}
\hline Technique & Knowledge transfer method \\
\hline Interview (unstructured) & Documentation - Storytelling \\
\hline Interview (Structured) & Documentation - Storytelling \\
\hline Task analysis & Documentation \\
\hline Card Sorting & Documentation \\
\hline Surveys & Documentation - Storytelling \\
\hline Protocol analysis & Documentation \\
\hline Repertory grid & Documentation - Legacy Development Plans \\
\hline Brainstorm & Documentation - Storytelling \\
\hline Nominal Group Technique & Documentation - Job Shadowing \\
\hline Observation & Documentation - Lessons Learned \\
\hline Prototyping & Documentation - Storytelling \\
\hline Focus Groups & Documentation - Education and Training \\
\hline JAD workshop & Documentation - Education and Training \\
\hline Scenario analysis & \\
\hline & \\
\hline &
\end{tabular}




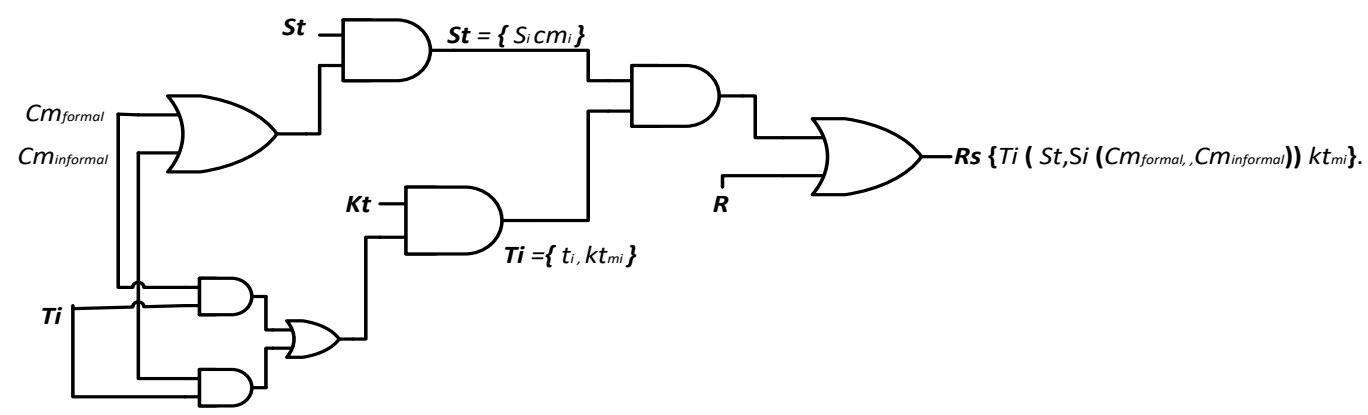

Figure 2. Communication model multiplexer

Some elicitation technique can not employ communication modes e.g., task analysis and survey technique, in this case the model needs to dictate the inputs so it does not select any of the communication mode parameters, hence the model employs a multiplexer with technique $\boldsymbol{T i}$ as a selector for the two inputs of communication mode $\boldsymbol{C m}$ (Cmformal, ,Cminformal), depending on the value of the $\boldsymbol{T} \boldsymbol{i}$ the multiplexer can only select the techniques that are communicative in nature. The model will direct the system analyst $\boldsymbol{S t}$ to specify the optimal method to initiate the two way communication with stakeholder $\{\boldsymbol{S i}\}$ with the specified reference of the communication mode $\{\boldsymbol{C m i}\}$ and the technique $\boldsymbol{T i}$ will be set all the its corresponding knowledge transfer method $\boldsymbol{K} \boldsymbol{t}$ as its been showed in the Table $2, \boldsymbol{T} \boldsymbol{i}=\{t i, k t m i\}$ is for every technique to operate through a specific knowledge transfer method to make the most of requirement elicitation and to acquire a complete requirement frame. As such $\boldsymbol{S t}=\{S i c m i\} \boldsymbol{A N D} \boldsymbol{T i}=\{t i, k t m i\}$ to be an input $\boldsymbol{O R}$ with the $\boldsymbol{R}$. The logic behind that is probability of encountering resistance.

\section{F. Phase \#2 Conflicts detection \& Resolution}

Requirements are information's. The system analysts already have a perceptual concept on the desired system, but they still keep in their mind that they are building a system that suites the stakeholders wishes, as such they value their stakeholder's involvement. The transformation of requirement to values starts at the beginning of the project, where the system analyst designs the main elements of the system as the following example of billing system:

Requirement: Analyst: Stakeholder: \{Data Input and Verification, system functionality, Tracking Payments, Security, Interface, Storage, Data Output $\} . \boldsymbol{R} x: \boldsymbol{S t} x: \boldsymbol{S} x\{c 1, c 2, . ., c n\}$

This template will be amended during the elicitation process to unify the requirements pattern, and the sub elements will be considered as classes that represent the system. As its been illustrated above the analyst already has a perceptional system design in which he or she sees fit based on the project goals and objectives, as such the model will rely on the analyst judgment to evaluate these 
classes based on their convincing argument, the classes will have a percentile values requirements output given by the analyst for each class based on the analyst judgment as follows:

Requirement 1: analyst 1: stakeholder1: $\{\boldsymbol{c} 1-90 \%, \boldsymbol{c} 2-70 \%, . ., \boldsymbol{c} n\}$

Requirement 1: analyst 2: stakeholder2: $\{\boldsymbol{c} 1-90 \%, \boldsymbol{c} 2-40 \%, . ., \boldsymbol{c} n\}$

Requirement 1: analyst 3: stakeholder3: $\{\boldsymbol{c} 1-80 \%, \boldsymbol{c} 2-60 \%, . ., \mathrm{cn}\}$

Requirement 2: analyst 1: stakeholder1: $\{\boldsymbol{c} 1-40 \%, \boldsymbol{c} 2-10 \%, . ., \mathrm{cn}\}$

Requirement 2: analyst 2: stakeholder2: $\{\boldsymbol{c} 1-50 \%, \boldsymbol{c} 2-40 \%, . ., \boldsymbol{c n}\}$

Requirement 2: analyst 3: stakeholder3: $\{\boldsymbol{c} 1-88 \%, \boldsymbol{c} 2-60 \%, . ., \boldsymbol{c} n\}$

Every requirement output will have the $\mathrm{R} x: \mathrm{St} x: \mathrm{S} x\{\mathrm{c} 1, \mathrm{c} 2, . ., \mathrm{cn}\}$ values for variable number of stakeholders, these values represent the elicited classes. These classes represent a small portion of the desired system; this pattern will be established for all the elicitation techniques, to allow the model to compare these classes separately and individually.

The model starts to calculate the correlation for the requirements sets of the same elicitation cases that has different contributors such as system analysts $\boldsymbol{S t}$ and stakeholders $\boldsymbol{S i}$ using the normalized cross correlations function (NCCF). As such Requirement 1 set will be represent three different system analyst elicitation case as follows: $\boldsymbol{S t x}$, $\boldsymbol{S t y}$ and $\boldsymbol{S t z}$.

a. The normalized cross correlations function (NCCF)

$$
\text { Corrxy } x \otimes y=\frac{\sum_{\mathrm{n}=0}^{\mathrm{N}-1} \operatorname{stx}[c] S t y[c]}{\sqrt{\sum_{\mathrm{n}=0}^{\mathrm{N}-1} \operatorname{St} x^{2}[c] \sum_{\mathrm{n}=0}^{\mathrm{N}-1} S t y^{2}[c]}}
$$

Equation 1: The normalized cross correlations function (NCCF)

The function will start to calculate the correlation between the classes $\boldsymbol{c}$ set inStx $\otimes S t y$, classes set in $\boldsymbol{S t} x \otimes \boldsymbol{S t z}$ and the classes set in $\boldsymbol{S t y} \otimes \boldsymbol{S t z}$. As a result the requirements sets with such a higher similarity value will be classified as a conflict free requirement due to the resemblance of the requirements content. The requirements set with lower similarity values will be processed for an in depth inspection using the Standard error (SE) function to calculate the margin error between the elicited requirements sets classes. In order to get the Standard error (SE) value, the model will need to calculate the Standard deviation (SD) values of the requirements sets.

\section{b. Standard deviation (SD)}

The formula for the sample standard deviation is: 


$$
\sigma=\sqrt{\frac{\sum_{i=1}^{N}(c i-\bar{c})^{2}}{N-1}}
$$

Equation 2: Standard deviation (SD)

Where $\{\mathbf{c 1} 1, \mathbf{c 2}, . . \mathbf{c n}\}$ of the $\mathbf{R} x: \mathbf{S t} x: \mathbf{S} x$ are the observed values of the sample items, $\overline{\boldsymbol{c}}$ is the mean value of these observations, and $\boldsymbol{N}$ is the number of observations in the sample and $\boldsymbol{\sigma}$ is the standard deviation, which will be calculated for the classes of the same requirement dataset as the following pattern:

$\left.\begin{array}{lcc}\begin{array}{l}\text { Requirement 1: analyst 1: stakeholder1: } \\ \{\mathbf{c} 1\}\end{array} & \{\mathbf{c} 1\} \\ \{\mathbf{c} 2\} & \text { set } 1\end{array}\right\}$ set 1

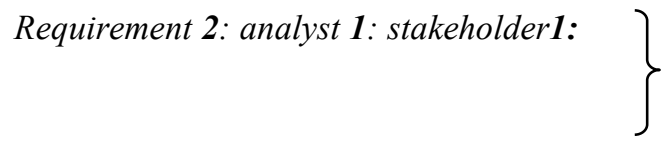

Requirement 1: analyst 2: stakeholder2: $\{\mathbf{c} 1\}\}$ $:\{\mathbf{c} 1\}$

set 2

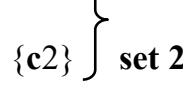

Requirement 2: analyst 2: stakeholder2



$\{\mathbf{c} n\}$

$\{\mathbf{c} n\}$

Requirement 1: analyst 3: stakeholder3: $\{\mathbf{c} 1\}$ $\{\mathbf{c} 1\}$

set 3

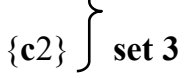

Requirement 2: analyst 3: stakeholder3:

$\{\mathbf{c} 2\}$

$\{\mathbf{c} n\}$

$\{\mathbf{c} n\}$

c. Standard Error (SE)

Can be expressed as:

$$
\mathrm{SE}_{\overline{\mathbf{c}}}=\frac{\sigma}{\sqrt{\mathbf{n}}}
$$

Equation 3: Standard Error (SE)

Where $\boldsymbol{\sigma}$ is the standard deviation of the population is the size (number of observations) of the sample. 
Standard error will be calculated for the requirements sets with lower correlation values, to measure their accuracy and validity. As such the requirements set which has a lower Standard error (SE) values will be considered partially conflicted therefore it will put into Trace source for validation phase, to trace the source of the requirement "the stakeholder" to validate the requirements, of course the trace will be on the conflicted classes of the requirements sets. As result the model will amend the new requirement sets and update the final requirements report.

\section{Conclusion}

The introduction section has illustrated the major issues facing the requirement elicitation process, communication obstacles and requirement conflicts, which leads to catastrophic results in term of projects budgets and delivery. This paper presents a resolution to elevate these project success rate ratios. The proposed model tackles the issues of communication barriers represented in articulation problem, by provides smooth transition of requirement through the use of specific knowledge transfer modes. The model resistance resolution protocol enhanced the communication channels and assured the stakeholder involvement and contribution to the intended system. The model conflicts detection \& resolution mechanism relies on the normalized cross correlations function (NCCF) to identify the similar requirement classes' taken from the raw requirement; this step is to define the similarity patterns within requirement classes and identifies conflict patterns. Furthermore, the model runs the Standard deviation (SD) and Standard error (SE) functions to measure the requirements accuracy and validity. The model will disqualifies the conflicted requirements to be considered as disruptive factors and noise with a higher potentiality risk contribution to drive the project toward failure. The used model and design will help the system analyst in the course of the requirement elicitation process to efficiently interact with the corresponding stakeholder and create higher standard shared knowledge requirements that are complete, appropriate, clear, and a conflict free representation of the desired system. As such the model will help in increasing the success ratio of the IT projects.

\section{References}

[1] All, A. (2009, December 22). Failed IT Projects Cost Way Too Much, No Matter How You Crunch the Numbers. Retrieved November 10, 2010, from IT Business Edge.

[2] Agarwal, R. \& Tanniru, M.R. "Knowledge Acquisition Using Structured Interviewing: An Empirical Investigation," Journal o f M anagem ent Information Systems (7:1),1990, pp. $123-140$

[3] Bostrom, R. P. (1989). Successful application of communication techniques to improve the systems development process. Information and Management, 16(5), 279-295.

[4] Lyytinen K, Hirschheim R. Information system failures - a survey and classification of the empirical literature. Oxford Surveys in Inform Tech 1987; 4: 257-309

[5] Macaulay LA. Requirements Engineering. Springer-Verlag, London, 1996

[6] Bourque, P., \& Fairley, R. E. (2014). Guide to the Software Engineering Body of Knowledge (SWEBOK (R)): Version 3.0. IEEE Computer Society Press 
[7]Tan, M. "Establishing Mutual Understanding in Systems Design: An Empirical Study," Journal o f M anagement Information System s (10:4), 1994, pp. 159-182.

[8] Saiedian, H., \& Dale, R. (2000, April). Requirements engineering: making the connection between the software developer and customer. Information and Software Technology, 42(6), 419428.

[9] Hirschheim, R., Newman, M., 1988. Information Systems and User Resistance: Theory and Practice. Comput. J. 31, 398-408

[10] Joshi, K., 1991. A model of Users' perspective on change: the case of information systems technology implementation. MIS Q. 15, 229-242. http://dx.doi.org/10.2307/249384.

[11] Marakas, G.M., Hornik, S., 1996. Passive resistance misuse: Overt support and covert recalcitrance in IS implementation. Eur. J. Inf. Syst. 5, 208-219. http://dx.doi.org/10.1057/ejis.1996.26

[12] Fiedler, S., 2010. Managing resistance in an organizational transformation: A case study from a mobile operator company. Int. J. Proj. Manag. 28, 370-383. http://dx.doi.org/10.1016/j.ijproman.2010.02.004.

Ford, J.D., Ford, L.W., D'Amelio, A., 2008. Resistance to change: the rest of the story. Acad. Manag. Rev. 33, 362-377.

[13] Laumer, S., 2011. Why Do people reject technologies - a literature-based discussion of the phenomena "resistance to change". ECIS 2011 Proceedings. Information Systems and Managerial Psychology Research

[14] Rivard, S., Lapointe, L., 2012. Information technology Implementers' responses to user resistance: nature and effects. MIS Q. 36, 897-920.

[15] Sigala, M., 2013. Examining the adoption of destination management systems: An interorganizational information systems approach. Manag. Decis. 51, 1011-1036. http://dx.doi.org/10.1108/MD-11-2012-0800.

[16] Stallinger, F., \& Gr, P. (2001). System dynamics modelling and simulation of collaborative requirements engineering, 59, 311-321.

[17] A.M. Davis, The harmony in rechoirments, IEEE Software 15 (2) (1998) 6-8.

[18] E.J. Garrity, Synthesizing user centered and designer centered IS development approaches using general systems theory, Information Systems Frontiers 3 (1) (2001) 107-121.

[19] Coughlan, J., Lycett, M., \& Macredie, R. D. (2003). Communication issues in requirements elicitation: a content analysis of stakeholder experiences, 45, 525-537. https://doi.org/10.1016/S0950-5849(03)00032-6

[20] Silva, J.R., dos Santos, E.A., 2004. Applying Petri Nets to requirements validation.

In: ABCM Symposium. Series in Mechatronics, vol. 1, pp. 508- 517. 